\title{
VARIACIÓN DE LA TEMPERATURA EN PUNTA ARENAS (CHILE) EN LOS ÚLTIMOS 120 AÑOS.
}

TEMPERATURE VARIATIONS IN PUNTA ARENAS (CHILE) DURING THE LAST 120 YEARS.

Ariel Santana, Nicolás Butorovic ${ }^{2} \&$ Carlos Olave $^{2}$

\begin{abstract}
RESUMEN
Se presenta la serie de temperaturas de Punta Arenas correspondientes a 120 años, en un periodo que va desde 1888 hasta el 2007. Se analizan las variaciones que ha tenido este parámetro atmosférico a través de estos años, concluyéndose que han ocurrido periodos intercalados de alta y de bajas temperaturas en torno a su valor medio. Respecto a su tendencia y en referencia al valor promedio, es más bien estable, aunque si se considera una regresión lineal, ésta muestra un leve enfriamiento durante estos 120 años. Un análisis estacional de esta variable muestra algunos matices respecto al comportamiento anual. Se analizan por separado las series de medias de las máximas y de las mínimas mensuales y se hace su comparación con las medias por estación del año en cada uno de éstas. Finalmente se analizan los valores extremos mensuales que ha tenido la temperatura en la ciudad y se presentan los valores más frecuentes que éstas han tenido en este periodo, así como los valores récord alcanzados.
\end{abstract}

Palabras clave: Punta Arenas, temperatura.

\section{ABSTRACT}

A sequence of temperature records from Punta Arenas for the 120 year period 1888 - 2007 are presented. The analyses of how temperature has varied over the years has concluded that there have been periods when the temperature has been below and above the median. A linear regression performed on the data showed that the temperature has slightly cooled over the 120 years. From a seasonal analysis of temperature, there were variable intra-seasonal differences between years. Mean monthly means of maximum and minimum temperature are compared by station and year. The frequency, occurrence and extremes of monthly temperatures in Punta Arenas over the period are presented.

Key words: Punta Arenas, temperature.

$1 \quad$ Laboratorio de Climatología. Instituto de la Patagonia. UMAG. asa@umag.cl.

2 Centro de Estudios del Cuaternario y Fuego-Patagonia. CEQUA. 


\section{INTRODUCCIÓN}

La superficie de la ciudad de Punta Arenas, al igual que cualquier superficie del planeta está sometida a cambios energéticos cotidianos y sistemáticos, producto de la dinámica del planeta en el sistema solar que se encuentra. En este constante variar energético (día-noche; invierno-verano) el sistema busca un equilibrio que nunca encuentra y sólo lo logra en forma relativa. El resultado de esto queda de manifiesto en la oscilación de la temperatura a través del tiempo.

Los registros climáticos de la región de Magallanes se remontan al siglo XVIII con las observaciones hechas por el comandante de la fragata española "Santa María de la Cabeza", que vino a estudiar las aguas del estrecho de Magallanes. El periodo de mediciones de temperaturas corresponde a los meses de diciembre de 1785 a marzo de 1786, en Puerto del Hambre. Esta localidad se encuentra ubicada a unos 50 kilómetros al sur de Punta Arenas, por lo que no se puede esperar que los datos sean muy coincidentes con los obtenidos en la ciudad.

Posteriormente, en el año 1834, el comandante FitzRoy realizó algunas mediciones en Puerto del Hambre, Cabo Negro y San Gregorio. Ahora sabemos que estas localidades pertenecen a climas totalmente diferentes entre ellas, por lo que su comparación con datos actuales sirven sólo como una referencia aproximada de los valores de Punta Arenas.

Otros antecedentes históricos de importancia corresponden a la expedición francesa a bahía Orange (cabo de Hornos) en 1882 y que duró 11 meses. En ellos se tiene un registro muy detallado de todas las variables atmosféricas durante el periodo que duró esta expedición. No obstante, la ubicación de esta localidad en más de tres grados de latitud al sur de la ciudad, hacen imposible una comparación apropiada.

Las siguientes observaciones meteorológicas corresponden al entonces gobernador de la colonia de Punta Arenas, don Jorge Cristian Schythe y abarcan un periodo de 9 años, comprendidos entre 1854 y 1863. Estos registros carecen de regularidad en el tiempo y están interrumpidos en forma casi sistemática en las épocas más frías y, si bien constituyen un antecedente valioso para el conocimiento de las condiciones meteorológicas de esos años, carece de la regularidad suficiente en el tiempo como para concluir algún tipo de comportamiento del clima en las distintas estaciones del año.

A partir de 1887 comienzan los registros regulares de temperaturas y otras variables meteorológicas en Punta Arenas, con la llegada de los Salesianos, en julio de ese año. La orden Salesiana se distingue de otras de su género, por sus trabajos pedagógicos y científicos. Entre sus obras más importantes destacan "El clima de Punta Arenas a través de 31 años de observaciones" (Re 1929) y en donde se detalla el origen del Observatorio Monseñor Fagnano y "El clima de Punta Arenas. 21 años de observaciones meteorológicas" (Re 1945). Las mediciones se iniciaron en diciembre de 1887 y continúan hasta el presente. Sin embargo, a través del tiempo, este observatorio ha cambiado su ubicación y altura de registros sobre el nivel del suelo. La instalación del instrumental meteorológico en el centro de Punta Arenas, se hizo bajo la supervisión de Monseñor José Fagnano, prefecto apostólico de la parte chilena de la Patagonia. En 1908/09 se reinstalaron los instrumentos en una pequeña torre de $5 \mathrm{~m}$ por sobre los demás edificios de la época. Así se realizan los registros a uno $15 \mathrm{~m}$ s. n. s. Posteriormente este observatorio se traslada a lo que hoy día es el Colegio Salesiano San José.

Después de los Salesianos, otras instituciones iniciaron los registros regulares con fines muy particulares en cada caso. Con fines operativos, destacan la Fuerza Aérea de Chile en bahía Catalina, en la década de 1930 (Ojeda 1962)르, estación que fue trasladada en 1964 al aeropuerto Carlos Ibáñez del Campo, $24 \mathrm{~km}$ al norte de la ciudad y donde permanece hasta ahora; y la Armada de Chile desde 1940 (Anuarios meteorológicos). Con objetivos principalmente científicos, están las estaciones del Instituto de la Patagonia de la Universidad de Magallanes y de la Fundación CEQUA; con fines legales y de uso de recursos hídricos, la Dirección General de Aguas (DGA); con fines energéticos, la empresa de electricidad y la Empresa Nacional del Petróleo (ENAP). Actualmente son muchas las instituciones que hacen registros meteorológicos en la región, para lo cual han instalado un número

1 Ojeda, R. 1962. Breve ensayo sobre el clima y la dinámica del clima en Punta Arenas. Fza Aérea de Chile. Of. Meteor. De Chile. Mimeo. 33 pp. 
considerable de estaciones de registro automático que, sin duda, contribuirán de manera importante al conocimiento más detallado del clima en esta parte de Sudamérica.

Cabe mencionar también que durante la época del auge ganadero, la Explotadora Tierra del Fuego inició en el año 1924 mediciones en forma simultánea de temperatura y precipitación en gran parte de sus estancias, obteniéndose una base de datos importante que a través de estudios posteriores han entregado un esquema bastante claro de los tipos climáticos de Magallanes. Esas mediciones continuaron, en algunos casos hasta 1966, fecha en que se produjo la reforma agraria y a partir de la cual, no se hicieron mas mediciones.

La estación Jorge C. Schythe del Instituto de la Patagonia, ubicada a $4 \mathrm{~km}$ al norte del centro de la ciudad de Punta Arenas, casi frente a la que fuera estación de bahía Catalina, se pone en marcha en 1970. Esta estación fue creada con fines netamente científicos y para hacer mediciones que no estuvieran afectadas por factores urbanos (Zamora 1977), continuando sus registros hasta el presente.

Actualmente se cuenta con un número mayor de estaciones de registros en variadas localidades de la región y a la luz de estos antecedentes se puede concluir que las variaciones de la temperatura en Magallanes son muy similares, al menos desde los $52^{\circ} \mathrm{S}$ hacia el sur, debido a la alta correlación que muestra esta variable en su variación en relación a sus promedios diarios (Schneider et al. 2003, Santana et al. 2005, Santana et al. 2007). Esto último es muy importante ya que al establecerse algún tipo de tendencia térmica en Punta Arenas, ésta puede ser inferida también para otras localidades.

Por otra parte las series largas de tiempo son escasas en el hemisferio sur, y la acumulación de antecedentes térmicos de más de un siglo representa una valiosa información, la cual puede entregar una visión clara de la tendencia de esta variable a través del tiempo. De esta manera ha sido posible completar una serie de temperaturas para Punta Arenas que alcanza a los 120 años, período suficiente como para observar las variaciones que ha tenido esta variable a través del tiempo, y visualizar la posibilidad de detectar los efectos de los eventuales cambios globales o alguna evidencia de un calentamiento global.

\section{MATERIAL Y MÉTODOS}

Las series utilizadas en el análisis corresponden a datos obtenidos de numerosas publicaciones del Observatorio Monseñor Fagnano (Re 1929,1945), datos de la estación de bahía Catalina (Ojeda 1962), de la estación Jorge Schythe del Instituto de la Patagonia (Zamora 1977, Zamora \& Santana 1978, 1980; Santana 1980, 1981, 1982, 1983, 1984, 1985, 1986, 1987, 1988, 1989, 1990, 1991, 1992, 1993, 1994, 1995, 1996, 1997, 1998, 1999, 2005, 2006, 2007, Butorovic 2000, 2001,2002, 2003, 2004), de la radio estación naval (Anuarios meteorológicos) y para comparación con el área de la ciudad, se utilizaron datos de los últimos 16 años del aeropuerto presidente Carlos Ibáñez del Campo.

Algunos autores afirman que la serie de Punta Arenas no es válida si es que no es sometida a una homogenización de sus datos (Rosenbluth et al. 1995). Existen dos formas de abordar el problema de la homogenización de los datos. La primera consiste en chequear exhaustivamente los registros y la segunda forma consiste en utilizar las inconsistencias con respecto a estaciones vecinas como guía para las in-homogeneidades mayores en la base de datos. Para esto último no se dispone de una serie de esa longitud que sirva como referencia.

En la homogenización de los datos se comprobó que la variación por cambios de altura sobre el suelo es prácticamente nula. Cuando se habla de la homogeneidad de una serie climatológica se entiende que se refiere a una homogeneidad relativa, es decir, en relación a las variaciones del elemento climático en la zona donde se ubica la estación, ya que en este tipo de series no se debe esperar homogeneidad absoluta en sentido puramente estadístico, o dejarían de tener sentido los trabajos sobre variabilidad y el cambio climático (Guijarro 2004). El estudio de la homogeneidad de una serie implica por tanto la comparación de la misma con una serie de referencia. Para ello se podría emplear una serie próxima de reconocida calidad y homogeneidad pero, como a priori es muy aventurado hacer esas suposiciones, resulta más conveniente usar una serie artificial, combinando varias series reales, con objeto de diluir sus propias posibles inhomogeneidades.

En el caso de Punta Arenas no es aplicable ninguna de las formas de homogenización mencio- 


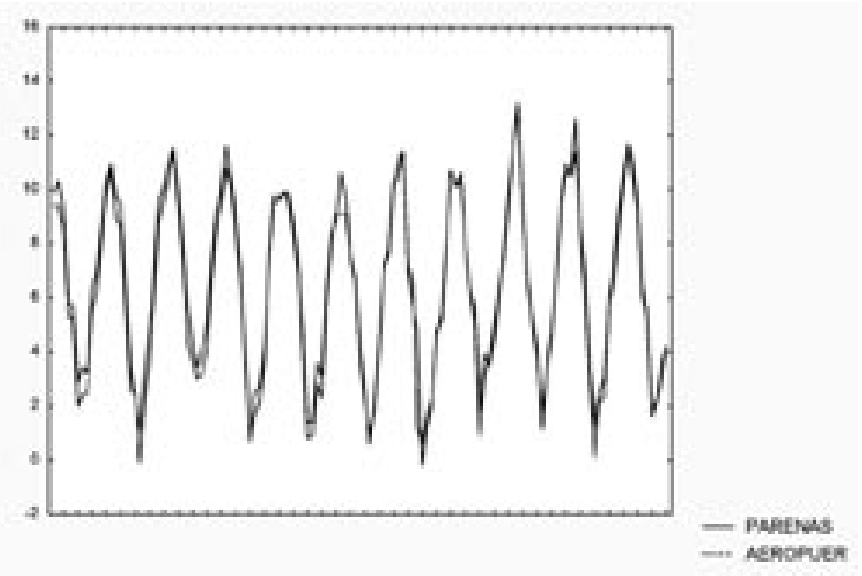

Fig. 1. Comparación de la temperatura media mensual en el aeropuerto Carlos Ibáñez y en Punta Arenas.

nadas anteriormente, ya que no existe una estación cercana con un registro similar y obtener un promedio de estaciones más cercanas es aún mas inaplicable debido a la gran distancia que las separa. En relación a la variación horizontal, ésta también se consideró mínima, puesto que los valores obtenidos tanto en la ciudad como en el aeropuerto Presidente Ibáñez, ubicado $24 \mathrm{~km}$ al norte de la misma, son prácticamente iguales tal como lo muestran los promedios mensuales de 16 años en ambas localidades en la figura 1. Esto hace suponer que en distancias menores (e.g. dentro del área de la ciudad), las diferencias son aún menores. Las pequeñas diferencias mostradas entre las curvas de la figura 1 se deben fundamentalmente a los métodos de obtener el valor medio mensual; mientras en Punta Arenas se utilizan cuatro valores para obtener una media diaria, en el aeropuerto ésta se calcula a partir de un valor cada hora.

Los cambios en la ubicación de la estación Fagnano (diferencias verticales) han sido mínimos lo cual produce diferencias imperceptibles. La variación horizontal por cambio de ubicación tampoco es importante y así lo demuestran los datos de la figura 1. Las distancias entre las estaciones consideradas aquí son significativamente menores, de manera que se puede inferir con propiedad que las diferencias entre estas son mínimas e inferiores al error humano que se puede cometer en la observación.

Para el cálculo de las anomalías anuales y estacionales de la temperatura, se utilizó la planilla Excel y la línea de tendencia se estableció mediante una exponencial simple, con lo cual se obtiene un comportamiento más suavizado de la variación de la temperatura a través del tiempo. Para el análisis estadístico también se utilizó el modulo TimeSID del paquete estadísticos internacional WinIDANS de la UNESCO.

\section{RESULTADOS Y DISCUSION}

Tras una revisión de los antecedentes disponibles, e.g. entre otros: Re (1929, 1945), Ojeda (1962), Zamora (1977), los valores que conforman la serie analizada consideran los antecedentes de casi todas las estaciones ya mencionadas. De esta manera, la tabla 1 muestra los valores medios, máximos y mínimos de temperatura para un periodo de 120 años de datos. Se homogenizaron los datos de acuerdo a la variación vertical y horizontalmente.

TABLA 1. Temperaturas medias y extremas para Punta Arenas 1888-2007.

\begin{tabular}{|c|c|c|c|c|c|c|c|c|c|c|c|c|c|}
\hline & Ene & $\mathrm{Feb}$ & Mar & Abr & May & Jun & Jul & Ago & Sep & Oct & Nov & Dic & Año \\
\hline Media & 11,0 & 10,6 & 8,8 & 6,5 & 4,0 & 2,2 & 1,8 & 2,8 & 4,6 & 6,9 & 8,7 & 10,2 & 6,5 \\
\hline Med. máx. & 15,0 & 14,6 & 12,8 & 9,9 & 6,7 & 4,7 & 4,3 & 5,5 & 7,9 & 10,7 & 12,6 & 14,1 & 9,9 \\
\hline Med. mín. & 6,5 & 6,0 & 5,1 & 3,3 & 1,3 & $-0,1$ & $-0,3$ & 0,4 & 1,4 & 2,9 & 4,3 & 5,6 & 3,0 \\
\hline Máxima & 29,9 & 28,3 & 24,2 & 21,4 & 18,7 & 16,5 & 15,0 & 14,3 & 19,2 & 21,5 & 24,4 & 26,9 & 29,9 \\
\hline Mínima & $-3,4$ & $-2,6$ & $-4,8$ & $-7,6$ & $-9,8$ & $-16,4$ & $-13,4$ & $-10,0$ & $-9,0$ & $-5,0$ & $-4,9$ & $-2,6$ & $-16,4$ \\
\hline
\end{tabular}




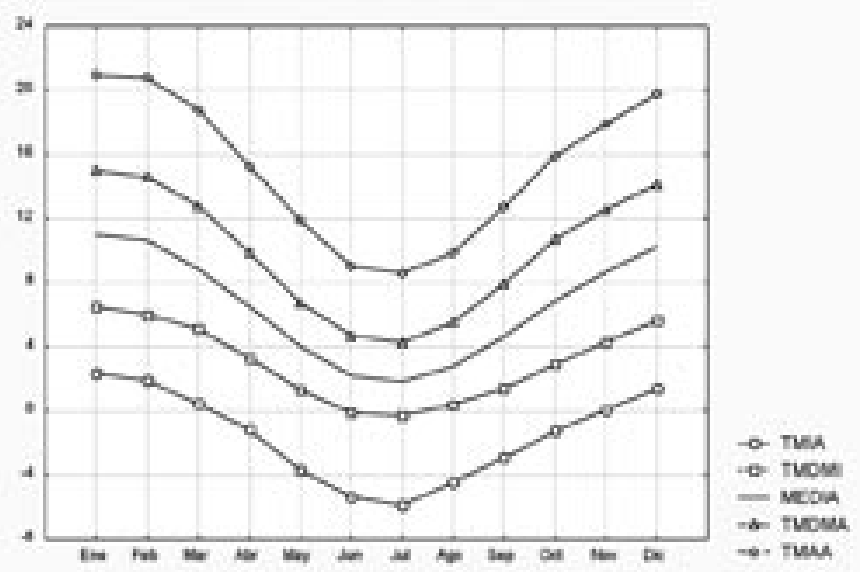

Fig.2. Promedio de la temperatura mensual en Punta Arenas en 120 años

TMIA=Temperatura promedio de las mínimas absolutas mensuales; TMDMI=Temperaturas medias de las mínimas diarias; MEDIA=Promedio mensual; TMDMA=Temperatura media de las máximas diarias; TMAA= Temperatura promedio de las máximas absolutas mensuales.

De acuerdo con la tabla 1 y a los 120 años de datos, la temperatura media para la ciudad de Punta Arenas es de $6,5^{\circ} \mathrm{C}$, valor idéntico al establecido en estudios anteriores (Endlicher \& Santana 1988, Zamora \& Santana 1989, Santana 2005, 2006) Los valores extremos oscilaron entre $29,9^{\circ}$ C, ocurrido en enero de 1900 y $-16,4^{\circ} \mathrm{C}$, ocurridos en junio de 1964.

En la figura 2 se muestra el comportamiento promedio mensual de la temperatura en los 120 años de análisis de los datos. De esta manera se puede ver que las temperaturas del mes más cálido, enero, presenta un promedio de $11^{\circ} \mathrm{C}$, con los promedios de las temperaturas extremas diarias entre $6^{\circ} \mathrm{C}$ y $15^{\circ} \mathrm{C}$ y con los promedios de las extremas absolutas entre $2^{\circ} \mathrm{C}$ y $21^{\circ} \mathrm{C}$. De la misma forma el mes mas frío presenta un promedio de $1,8^{\circ} \mathrm{C}$, con extremas diarias que fluctúan entre los $0^{\circ} \mathrm{C}$ y los $4^{\circ} \mathrm{C}$ y extremas mensuales entre los $-6^{\circ} \mathrm{C}$ como mínima y los $9^{\circ}$ C como máximas.

\section{Temperaturas medias}

Si se examina la serie de temperaturas medias anuales en los 120 años se observa una tendencia lineal negativa, es decir, las temperaturas estarían variando hacia un enfriamiento. Sin embargo, dentro del periodo analizado se observan épocas de calentamiento alternadas con épocas de enfriamiento (Zamora \& Santana 1979). De acuerdo a lo mostrado en la figura 3, se observa que estos periodos de calentamiento seguidos de periodos de enfriamiento son de diferente duración.

Un primer periodo de calentamiento se observa entre los años 1888 a 1901, con un incremento de $0,6^{\circ} \mathrm{C}$ en estos 14 años. En ese periodo la temperatura media osciló entre $5,9^{\circ} \mathrm{C}$ y $7,3^{\circ} \mathrm{C}$ con una excepción del año 1901 que alcanzó a 8,1 $\mathrm{C}$ y que corresponde a la media anual más alta de todo el periodo analizado. Otro período observable es el comprendido entre 1902 y 1906. En este quinquenio la temperatura descendió alrededor de $1,6^{\circ} \mathrm{C}$ y los promedios anuales fluctuaron entre $6,9^{\circ} \mathrm{C}$ y $5,1^{\circ} \mathrm{C}$. Este último valor corresponde al promedio anual más bajo de la serie de 120 años. Un segundo periodo de ascenso de temperaturas se inicia en 1907 y finaliza 24 años después, en

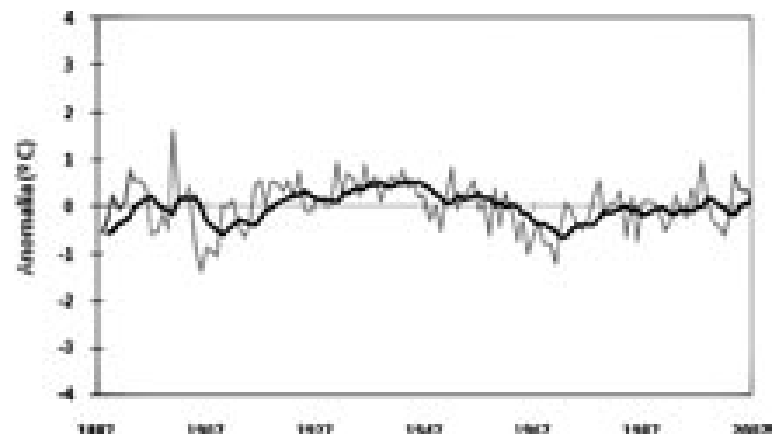

Fig. 3. Anomalías de la temperatura media anual en Punta Arenas. 
1931. En este periodo los promedios anuales de temperaturas oscilaron entre $5,5^{\circ} \mathrm{C}$ y $7,1^{\circ} \mathrm{C}$. A partir de 1932 y hasta 1971 la temperatura fluctuó entre $7,3^{\circ} \mathrm{C}$ y $5,3^{\circ} \mathrm{C}$, observándose un descenso más lento y estable que el anterior periodo de descenso de temperatura. Hasta aquí estos periodos están bien definidos en Zamora \& Santana (1979). Desde 1972 hasta el 2007 se aprecia un ascenso de la temperatura, que alcanza su máximo valor el año 1998 en que llegó a los 7,4 C como promedio anual. Las temperaturas en este tramo, oscilaron entre los $5,8^{\circ} \mathrm{C}$ (1986) y los ya mencionados $7,4^{\circ}$ C (1998). Aunque el promedio en este tramo fue de $6,5^{\circ} \mathrm{C}$, si se analiza los últimos 12 años, es decir, el periodo 1996 al 2007 el promedio es de $6,6^{\circ} \mathrm{C}$ y las tendencia se muestra prácticamente estable.

Como la temperatura no obedece a una función lineal en su comportamiento, es conveniente utilizar otra línea de tendencia que exprese mejor su variación. Para esto hemos utilizado una suavización exponencial simple y la curva que muestra esta variación se muestra en cada figura con color más oscuro y mayor espesor.

El gráfico de la figura 3 muestra las anomalías de la temperatura media a través de los 120 años de datos. Ahí se puede observar que la temperatura ha oscilado levemente en torno al valor medio ya establecido y existen periodos en que la temperatura estuvo por sobre la media y periodos donde estuvieron bajo este valor. No se observa una tendencia bien definida hacia un calentamiento o enfriamiento, sino más bien se observan periodos en que la temperatura tiende a ser mayor al promedio $y$ otros periodos con comportamiento contrario a este, bien representado por la línea suavizada. Con las anomalías se visualiza de mejor manera cuanto se separa del valor medio, la temperatura obtenida en un año cualquiera.

Una primera observación de la figura 3 muestra que la temperatura oscila levemente en torno a su valor medio, con variaciones máximas anuales que llegan a unos $1,9^{\circ} \mathrm{C}$ por sobre la media (1891) y a unos $1,4^{\circ} \mathrm{C}$ bajo el promedio (1901).

Si se calcula una tendencia lineal a la serie en estos 120 años, la línea que la define tiene una pendiente negativa, lo cual indica una tendencia lineal al enfriamiento. Sin embargo, una línea de este tipo no es concluyente puesto que es muy variable en función del largo del periodo a considerar. Sin embargo, existen algunas diferencias en este comportamiento cuando se lo analiza a nivel estacional. Así, en la estación de verano se aprecia una baja importante en la temperatura a fines de los años 60 y comienzos de los 70; variación, que si bien se presenta en las otras estaciones del año, no representa a las bajas más importante de la serie, como en el caso del verano.

Si se analiza cada estación a nivel de tendencia general, es la primavera la que muestra una tendencia al aumento de la temperatura en este periodo, aunque esa alza se manifiesta principalmente desde fines del siglo 19 hasta fines de la década de 1920. Por otra parte, en todas las estaciones del año quedan de manifiesto los altos promedios de temperaturas registradas en torno al año 1940.

La figura 4 muestra el comportamiento de la temperatura media en cada estación del año para el periodo 1888-2007.

De manera general se puede establecer que las temperaturas medias estacionales tuvieron una oscilación inferior a los $6,0^{\circ} \mathrm{C}$. La estación de verano es la que presentó las mayores anomalías con respecto a su promedio, llegando a registrarse valores de hasta $2,9^{\circ} \mathrm{C}$ bajo el promedio y de hasta $2,8^{\circ} \mathrm{C}$ por sobre éste. En las otras estaciones del año la oscilación térmica fue inferior. A nivel anual la oscilación llegó a $3^{\circ} \mathrm{C}$, alcanzándose anomalías de $1,6^{\circ} \mathrm{C}$ sobre el promedio y $1,4^{\circ} \mathrm{C}$ bajo éste. La tabla 2 muestra un resumen de los parámetros estadísticos de las temperaturas medias anual y por estación.

TABLA 2. Resumen estadístico de las temperaturas medias anual y estacional.

\begin{tabular}{|c|c|c|c|c|c|}
\hline & verano & otoño & invierno & primavera & Año \\
\hline media & 10,6 & 6,4 & 2,3 & 6,7 & 6,5 \\
\hline Desv. Estándar & 0,741 & 0,706 & 0,798 & 0,762 & 0,502 \\
\hline Mínimo & $-2,9$ & $-2,8$ & $-2,2$ & $-2,2$ & $-1,4$ \\
\hline Máximo & 2,8 & 1,7 & 1,8 & 2,2 & 1,6 \\
\hline
\end{tabular}




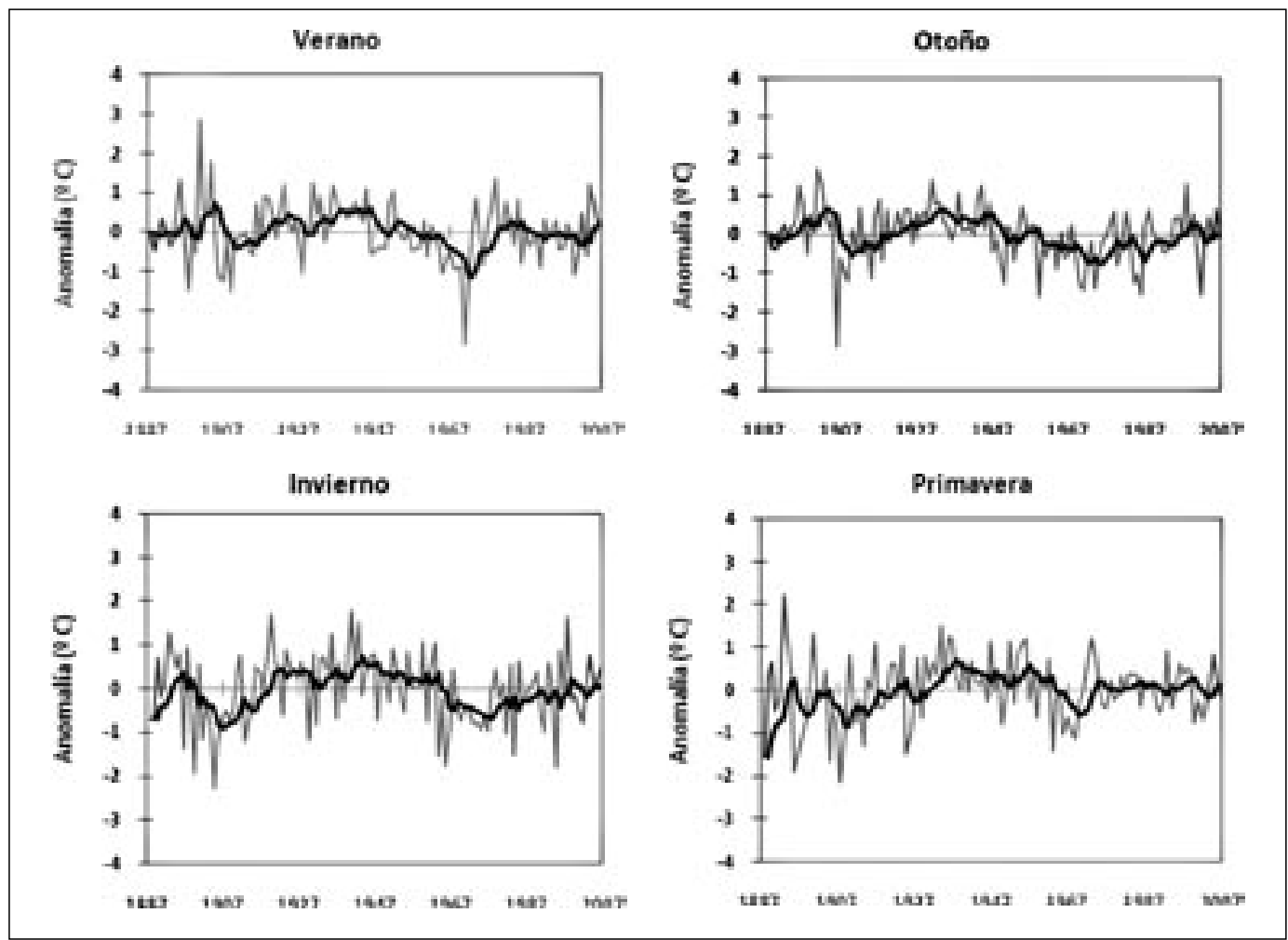

Fig. 4. Anomalías de la temperatura media por estación.

Temperaturas medias de las máximas

Las temperaturas medias de las máximas tienen un comportamiento que difiere del de las temperaturas medias. Al observar la serie de 120 años se aprecia claramente una tendencia al aumento de la temperatura media máxima conforme se avanza en los años, aunque también se observan periodos intermedios en los que la temperatura baja del promedio, como ocurre a fines de los años ' 60 y comienzos de los '70. Sin embargo, una estabilidad se muestra claramente entre los años 1945-1970. Al igual que en la temperatura media, los comportamientos estacionales muestran algunos matices respecto del comportamiento general. Así, para el año 1909, la anomalía mayor fue en primavera y alcanzó a $-4,5^{\circ} \mathrm{C}$ respecto de su promedio.

Otra anomalía negativa importante ocurrió en el promedio de verano del año 1971, con un valor de $2,7^{\circ} \mathrm{C}$ bajo su valor medio. Cabe señalar que esta anomalía negativa no se detecta claramente en las demás estaciones del año.
La figura 6 muestra el comportamiento de las temperaturas medias de las máximas para cada estación del año, junto a línea suavizada de tendencias general.

\section{Temperaturas medias de las mínimas}

El comportamiento de las temperaturas medias de las mínimas difiere de las dos series anteriores

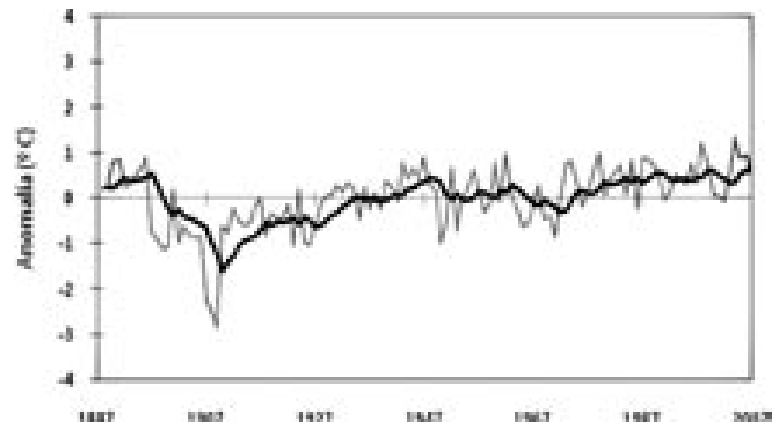

Fig. 5. Anomalías de la temperatura media de las máximas en Punta Arenas. 


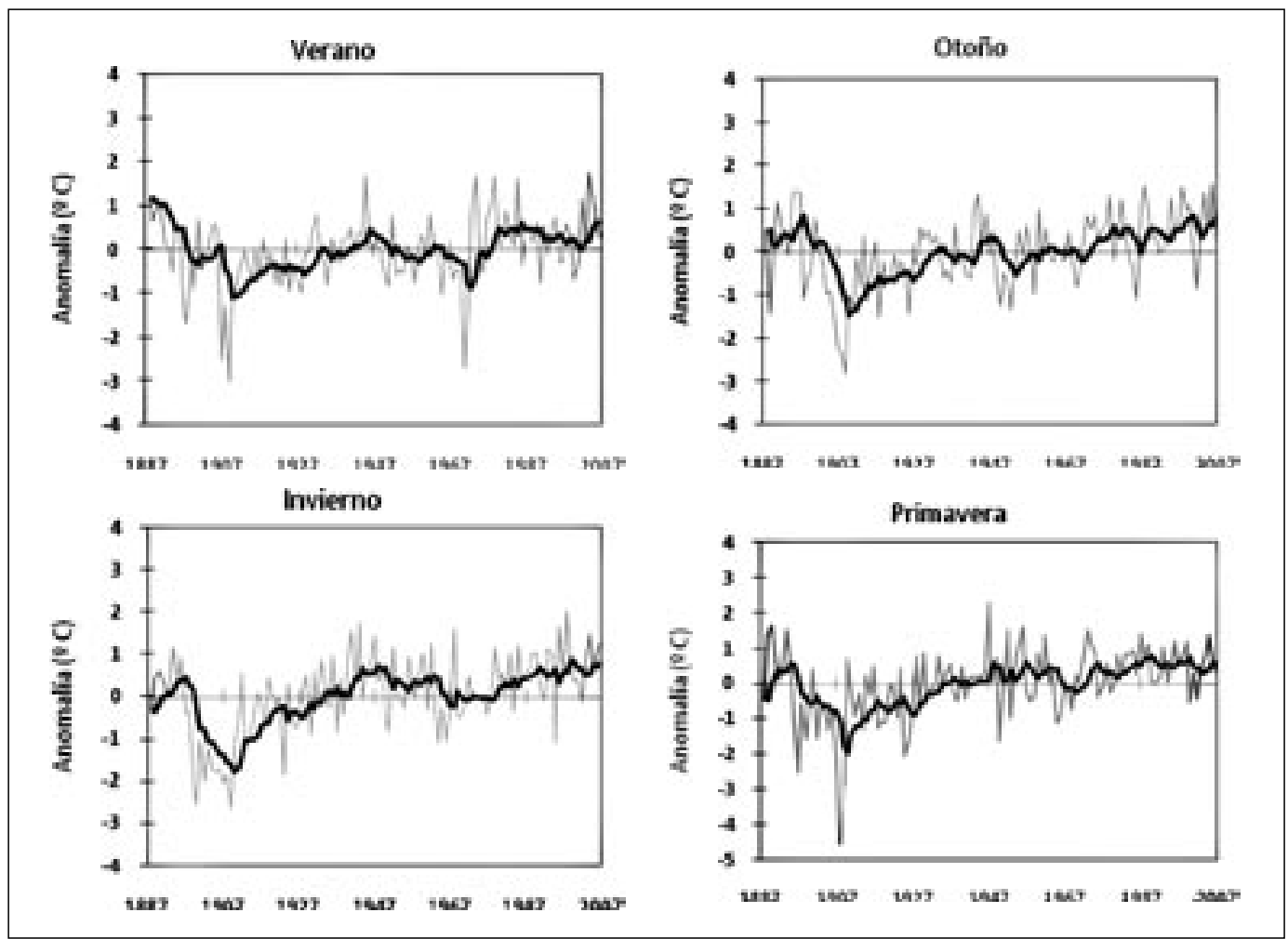

Fig. 6. Anomalías de la temperatura media de las máximas por estación.

y después de una alza sostenida de la temperatura desde 1888 hasta 1901, se observa una disminución que duró hasta 1912, donde se inicia un aumento de las mínimas que dura hasta 1924, año en que comienza una disminución de esta temperatura que concluye en 1986, completando un periodo de 62 años en que las mínimas fueron disminuyendo paulatinamente. A partir de ahí las mínimas nuevamente aumentan hasta el 2007, periodo en el

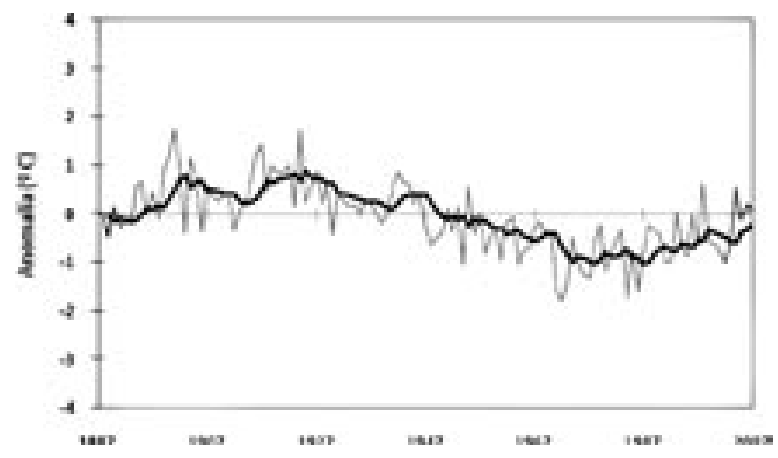

Fig. 7. Anomalías de la temperatura media de las mínimas en Punta Arenas. cual los promedios aún permanecen bajo la media histórica y lejos de los valores alcanzados entre los años 1890 a 1930.

El análisis estacional de esta variable también muestra algunas diferencias con el comportamiento general anual y la diferencia más evidente se observa en el comportamiento que tiene la estación de invierno en relación a las otras estaciones del año.

Mientras las estaciones muestran una especie de oscilación de la temperatura media de las mínimas desde comienzos del periodo analizado, en el cual la temperatura sube hasta alrededor de 1917 para posteriormente bajar paulatinamente hasta el año 1986 y posteriormente volver a subir hasta el 2007 (situación mejor expresada en el comportamiento de primavera) la estación de invierno muestra un descenso paulatino de la temperatura desde comienzos del periodo $y$, el que finaliza en 1984. A partir de este año se observa un aumento en estos promedios hasta 2007, aunque estos valores todavía permanecen bajo la media histórica y lejos aun de los altos promedios alcanzados en otros periodos. 


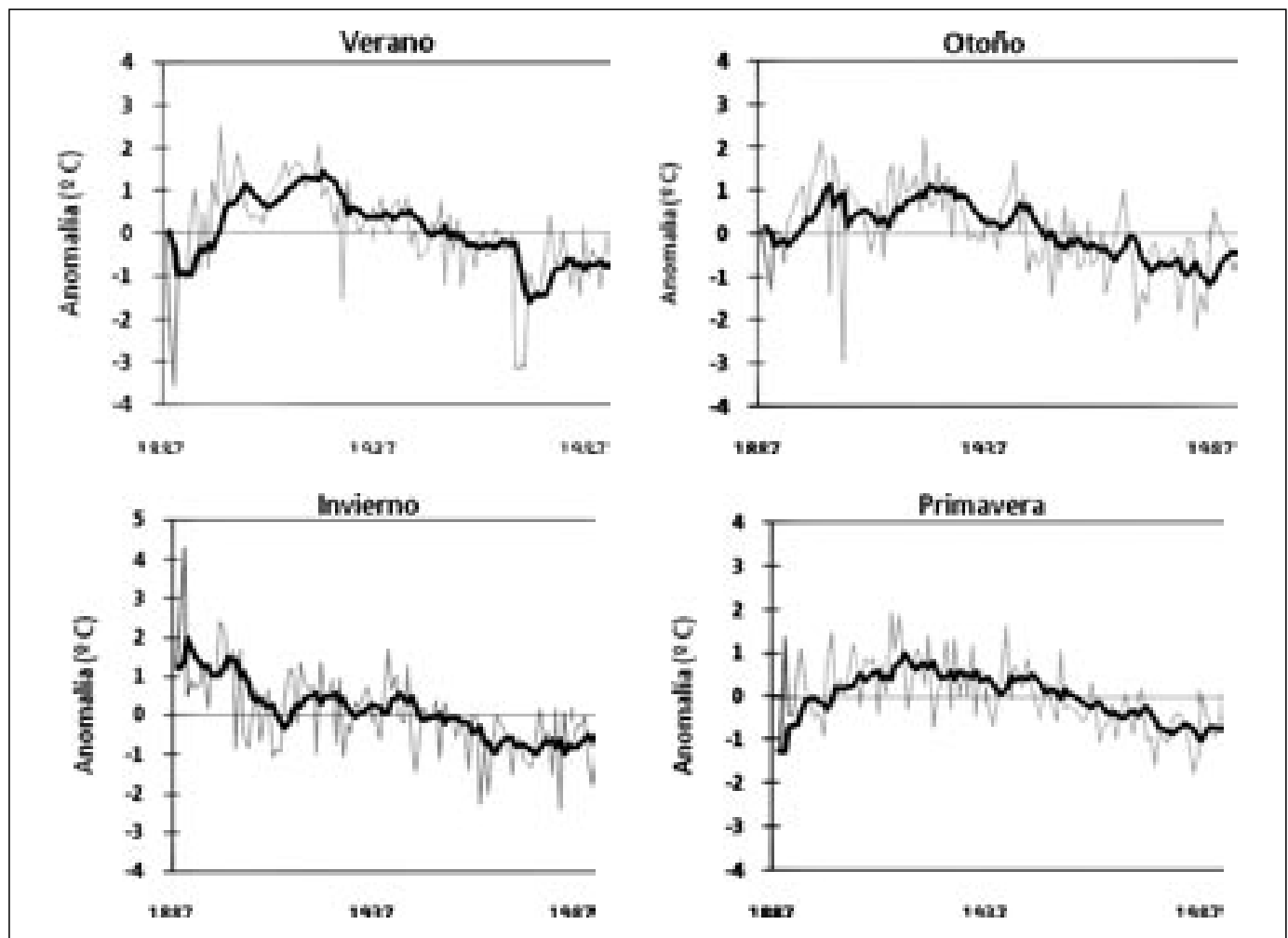

Fig. 8. Anomalías de la temperatura media de las mínimas por estación.

\section{Temperaturas extremas}

El comportamiento de las temperaturas extremas por su parte, no muestra grandes variaciones en estos 120 años. Las máximas registradas en Punta Arenas fluctúan entre $18^{\circ} \mathrm{C} y$ $30{ }^{\circ} \mathrm{C}$ (específicamente el límite superior llegó a $29,9^{\circ} \mathrm{C}$ registrados en enero de 1901 ), mientras que las mínimas oscilaron entre $-2,0^{\circ} \mathrm{C}$ y $-14,0^{\circ}$ C, con la excepción de 1964, que en julio de ese año registró $-16,4^{\circ} \mathrm{C}$, representando el récord del periodo. La figura 9 muestra el desarrollo que tuvieron las temperaturas extremas durante estos 120 años.

La figura 10 muestra los histogramas de frecuencia de ocurrencia de las temperaturas extremas. De las temperaturas máximas registradas, se observa que la mayor ocurrencia de ellas se encuentra entre los $20^{\circ} \mathrm{C}$ y los $27^{\circ} \mathrm{C}$, con mayor frecuencia entre $21^{\circ} \mathrm{C}$ y $22^{\circ} \mathrm{C}$. Las temperaturas mínimas por su parte, presentaron una mayor frecuencia entre $-4^{\circ}$ $\mathrm{C}$ y $-8^{\circ} \mathrm{C}$, con un máximo de ocurrencias entre $-6,0^{\circ} \mathrm{C}$ y $-7,0^{\circ} \mathrm{C}$.
Algunos autores, basados en que los datos de las series obtenidas con instrumentos meteorológicos no son confiables, han deducido el comportamiento térmico a partir de otras técnicas como la dendrocronología. Villalba et al. (2003), apoyado en informes de la OMM (1998), manifiesta la dificultad de basar los análisis en datos obtenidos instrumentalmente. Este autor señala que es muy difícil documentar a largo plazo el cambio de la temperatura durante

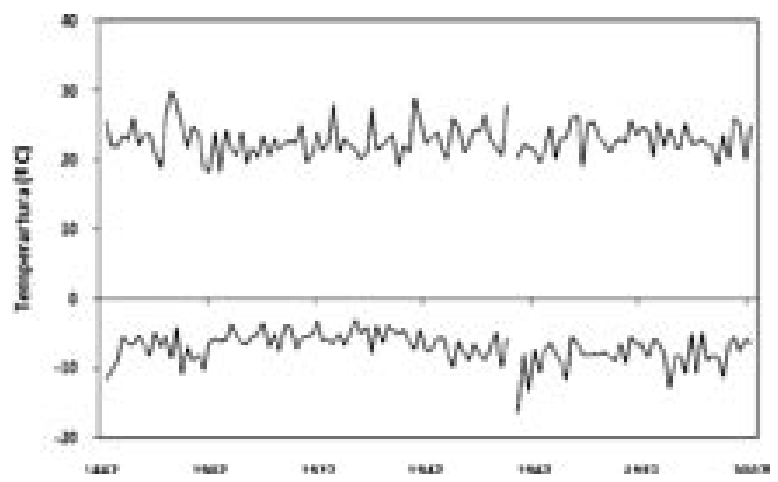

Fig. 9. Temperaturas máximas y mínimas mensuales en Punta Arenas. 


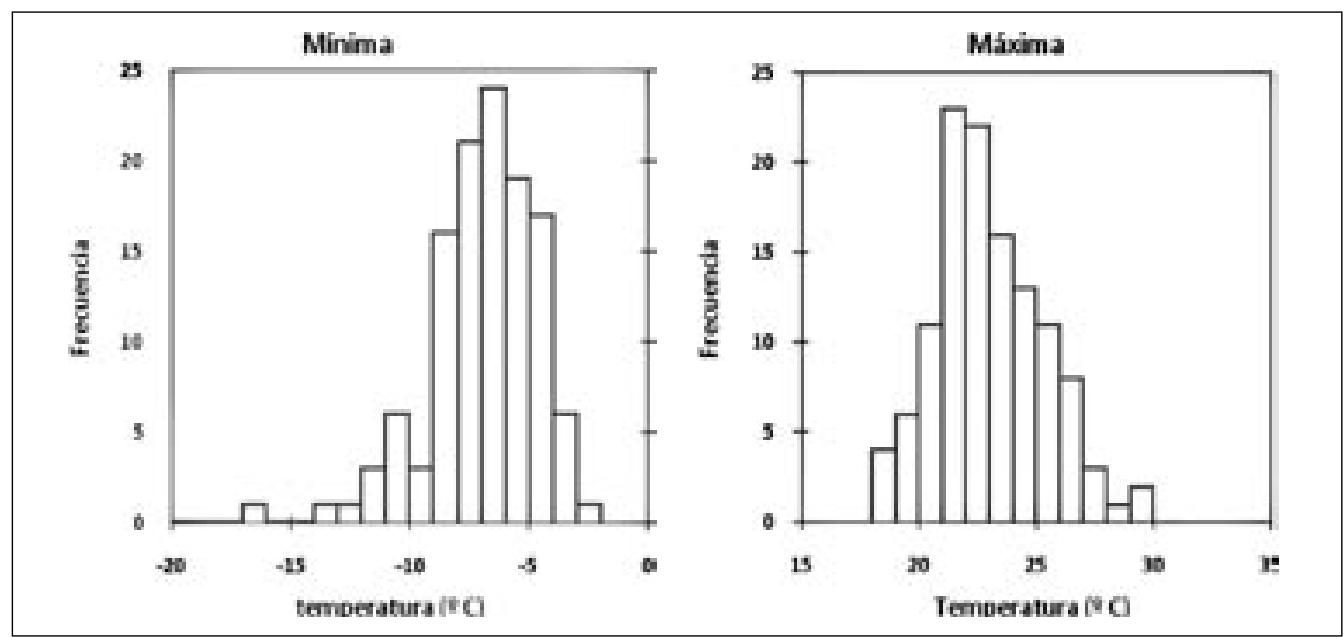

Fig. 10. Histogramas de las temperaturas extremas anuales en Punta Arenas.

el siglo 20 en las latitudes del sur $\left(40^{\circ}-60^{\circ} \mathrm{S}\right)$. Rosenblüth et al. (1997) argumenta que debido a la pobre calidad de los datos e instrumental, al escaso número de estaciones meteorológicas; que las series de tiempo son cortas, fragmentadas, con cambios indocumentados en instrumentación y ubicaciones de la estación, sumado a la existencia de muchos microclimas, hacen difícil realizar estudios completos sobre la variación de la temperatura en el hemisferio sur.

A partir de esto, algunos de esos y otros autores, han utilizado archivos paleo-ambientales para deducir una serie de temperaturas para Punta Arenas. De esta forma, Villalba et al. (2003) menciona que la temperatura del hemisferio ha aumentado en aproximadamente $0,3^{\circ} \mathrm{C}$ a $0,6^{\circ} \mathrm{C}$ desde fines del siglo 19 y en aproximadamente $0,2^{\circ} \mathrm{C}$ a $0,3^{\circ} \mathrm{C}$ desde mediado de los '70. La serie de temperaturas resultante de estos métodos resulta muy diferente a la mostrada por los datos instrumentales de la serie de Punta Arenas analizados aquí. Mientras estos autores muestran un excesivo calentamiento para Punta Arenas, los antecedentes presentados en este trabajo muestran una tendencia casi estable o hacia un leve enfriamiento. Por otra parte, la envergadura del proyecto Salesiano, los objetivos planteados, los fondos comprometidos, constituyen una garantía que los instrumentos adquiridos y utilizados en sus mediciones eran de primera calidad y carentes de grandes errores, por lo cual los errores posibles se pueden atribuir principalmente a fallas humanas en la observación. Hoy día se utiliza una variada gama de instrumentos y sensores para medir la temperatura ambiente, presentando diferencias mínimas entre ellos.

Son pocos los trabajos en que se puede encontrar series tan completas de temperaturas, similares a las analizadas aquí. Algunas series completas, a nivel hemisférico, muestran tendencias similares con la de Punta Arenas ${ }^{1}$.

\section{CONCLUSIONES}

Las variaciones tanto verticales como horizontales debido a cambios de posición y/o emplazamiento de las estaciones meteorológicas en Punta Arenas, son mínimas y no representan un inconveniente para un correcto análisis de los datos.

La temperatura en Punta Arenas ha variado en torno a una media de $6,5^{\circ} \mathrm{C}$ en periodos de calentamiento alternados por periodos de enfriamiento. Dichos periodos son de diferente duración. La tendencia general de toda la serie es casi estable, mostrando un leve enfriamiento.

Las temperaturas medias estacionales tuvieron una oscilación inferior a $6^{\circ} \mathrm{C}$ y fue la estación de verano donde ocurrieron las mayores anomalías.

Las temperaturas medias de las máximas tienen un comportamiento diferente al de las medias y de manera general muestran una tendencia al aumento.

1 http://www.rimfrost.no/ http://www.john-daly.com/index.htm 
Los comportamientos estacionales de las temperaturas medias de las máximas, al igual que las medias muestran algunos matices respecto al comportamiento anual.

Las temperaturas medias de las mínimas muestran un comportamiento que difiere de las dos series anteriores y después de un alza sostenida de 13 años, las mínimas muestran una disminución importante a través de 62 años de manera sostenida desde 1924 hasta 1986.

El análisis estacional de las mínimas muestra que en la estación de invierno, éstas no oscilaron como en las demás estaciones del año, sino más bien la temperatura descendió en forma casi continua durante los 120 años.

Las temperaturas extremas mensuales alcanzadas durante este periodo analizado no muestran grandes variaciones. La extrema máxima llegó a los $29,9^{\circ} \mathrm{C}$, mientras que la extrema mínima en la ciudad llego a los $-16,4^{\circ} \mathrm{C}$.

Las temperaturas máximas mensuales más frecuentes están entre $21^{\circ} \mathrm{Cy} 22^{\circ} \mathrm{C}$, mientras que las mininas mensuales ocurren entre $-6^{\circ} \mathrm{C}$ y $7^{\circ} \mathrm{C}$.

$\mathrm{Al}$ analizar las tres series de temperatura (media, media-máx., media-mín.), todas presentan tendencias diferentes; mientras la temperatura media muestra tendencia casi estable con una leve inclinación al enfriamiento, la media de las máximas tiende al aumento, en tanto que la media de las mínimas lo hace al enfriamiento. Esto lleva a concluir que la amplitud térmica a través de los períodos analizados está aumentando aunque esto no se detecta claramente cuando se analizan las temperaturas extremas y temperaturas medias estacionales.

Las serie de temperatura de Punta Arenas, deducida a partir de métodos distintos a los datos obtenidos instrumentalmente presentan diferencias importantes, de manera tal que las tendencias generales entre ambas series es contraria.

\section{LITERATURA CITADA}

Butorovic, N. 2001. Resumen Meteorológico año 2000. Estación Jorge C. Schythe. Anales Instituto Patagonia, Serie Cs. Nat. (Chile) 29:173-182.

Butorovic, N. 2002. Resumen Meteorológico año 2001. Estación Jorge C. Schythe. Anales Instituto Patagonia, Serie Cs. Nat. (Chile) 30:157-164.
Butorovic, N. 2003. Resumen Meteorológico año 2002. Estación Jorge C. Schythe. Anales Instituto Patagonia, Serie Cs. Nat. (Chile) 31:123-130.

Butorovic N. 2004. Resumen Meteorológico año 2003. Estación Jorge C. Schythe. Anales Instituto Patagonia (Chile), 32: 79-86.

Butorovic N. 2005. Resumen Meteorológico año 2004. Estación Jorge C. Schythe. Anales Instituto Patagonia (Chile), 33: 65-71.

Endlicher W. \& A. Santana 1988. El clima del sur de la Patagonia y sus aspectos ecológicos. Un siglo de Mediciones Climatológicas en Punta Arenas. Anales Instituto Patagonia, Serie Cs. Nat. (Chile), 18:57-86

Guijarro J., 2004. CLIMATOL: Software libre para la depuración y homogeneización de datos climatológicos. García Codron, J.C.; Diego Liaño, C.; Fdez. De Arróyabe Hernández, P.; Garmendia Pedraja, C. y Rasilla Álvarez, D. (Eds.) (2004). El Clima entre el Mar y la Montaña. Asociación Española de Climatología y Universidad de Cantabria, Serie A, $N^{\circ} 4$, Santander.

Re, José S. S. 1920. El clima de Punta Arenas. 32 años de observaciones meteorológicas (1888-1918) Observatorio Meteorológico José Fagnano. Punta Arenas. Chile, 89 pp.

Re, José S. S. 1945. El clima de Punta Arenas, 21 años de observaciones meteorológicas. 1919-1940. Observatorio Meteorológico José Fagnano. Punta Arenas. Chile, 132 pp.

Rosenblüth, B., G. Cassasa; H. Fuenzalida. 1995. Recent climatic changes in western Patagonia. Bulletin of Glacier Research 13 (1995) 127:132.

Rosenbluth, B., H. Fuenzalida \& P. Aceituno 1997: Recent Temperatura Variations in South America. Internacional Journal of Climatology, Vol. 17, 67-85.

Santana, A. 1982. Resumen Meteorológico año 1982. Estación Jorge C. Schythe. Anales Instituto Patagonia, (Chile) 13:225-230.

Santana, A. 1983. Resumen Meteorológico año 1983. Estación Jorge C. Schythe. Anales Instituto Patagonia, (Chile) 14:133-138.

Santana, A. 1984. Resumen Meteorológico año 1984. Estación Jorge C. Schythe. Anales Instituto Patagonia, (Chile) 15:61-66. 
Santana, A. 1985-1986. Resumen Meteorológico año 1985. Estación Jorge C. Schythe. Anales Instituto Patagonia, Serie Cs. Nat. (Chile) 16:91-98.

Santana, A. 1987. Resumen Meteorológico año 1986. Estación Jorge C. Schythe. Anales Instituto Patagonia, Serie Cs. Nat. (Chile) 17:103-109.

Santana, A. 1988. Resumen Meteorológico año 1987 y 1988. Estación Jorge C. Schythe. Anales Instituto Patagonia, Serie Cs. Nat. (Chile) 18:127-142.

Santana, A. 1989-1990. El Clima de Punta Arenas Durante 1989 y 1990. Anales Instituto Patagonia, Serie Cs. Nat. (Chile) 19:81-91.

Santana, A. 1991. El Clima de Punta Arenas Durante 1991. Anales Instituto Patagonia, Serie Cs. Nat. (Chile) 20:113-123.

Santana, A. 1992. El Clima de Punta Arenas Durante 1992. Anales Instituto Patagonia, Serie Cs. Nat. (Chile) 21:147-157.

Santana, A. 1994. Resumen Meteorológico año 1993. Estación Jorge C. Schythe. Anales Instituto Patagonia, Serie Cs. Nat. (Chile) 22:79-86

Santana, A. 1995. Resumen Meteorológico año 1994. Estación Jorge C. Schythe. Anales Instituto Patagonia, Serie Cs. Nat. (Chile) 23:119-126.

Santana, A. 1996. Resumen Meteorológico año 1995. Estación Jorge C. Schythe. Anales Instituto Patagonia, Serie Cs. Nat. (Chile) 24:97-104.

Santana, A. 1997. Resumen Meteorológico año 1996. Estación Jorge C. Schythe. Anales Instituto Patagonia, Serie Cs. Nat. (Chile) 25:127-134.

Santana, A. 1998. Resumen Meteorológico año 1997. Estación Jorge C. Schythe. Anales Instituto Patagonia, Serie Cs. Nat. (Chile) 26:141-149.

Santana, A. 1999. Resumen Meteorológico año 1998. Estación Jorge C. Schythe. Anales Instituto Patagonia, Serie Cs. Nat. (Chile) 27:117-124.

Santana, A. 2000. Resumen Meteorológico año 1999. Estación Jorge C. Schythe. Anales Instituto Patagonia, Serie Cs. Nat. (Chile) 28:143-150.

Santana A. 2006. Resumen Meteorológico año 2005. Estación Jorge C. Schythe. Anales Instituto Patagonia (Chile), 34: 81-90.
Santana, A., C. Porter, N. Butorovic, C. Olave. 2006. Primeros antecedentes climatológicos de estaciones automáticas (AWS) en el Canal Beagle, Magallanes, Chile. Anales Instituto Patagonia (Chile), 34: 5- 20.

Santana A. 2007. Resumen Meteorológico año 2006. Estación Jorge C. Schythe. Anales Instituto Patagonia (Chile), 35(1): 81-89

Santana, A., C. Porter, N. Butorovic, C. Olave 2007. Características climáticas del Canal Brecknock en los 54 30'de latitud, Magallanes, Chile. Anales Instituto Patagonia (Chile), 35 (1): 5- 18.

Santana, A. 2008. Resumen Meteorológico año 2007. Estación Jorge C. Schythe. Anales Instituto Patagonia (Chile). 36(1): 79-87.

Schneider, C., M. Glaser, R. Kilian, A. Santana, N. Butorovic, G. Cassasa 2003. Regional Climate Variations across the Southern Andes at $53^{\circ} \mathrm{S}$. Physical Geography. Vol. 24:97-119.

Villalba, R., A. Lara, J. A. Boninsegna, M. Masiokas, S. Delgado, J. C. Aravena, F. A. Roig, A. Schmelter, A. Wolodarsky, \& A. Ripalta 2003. Large-scale temperature changes across the Southern Andes: 20th century variations in the context of the past 400 years. Climatic change 59, 177-232.

Zamora, E. 1977. Resumen Meteorológico 1972-1977. "Estación Jorge CH. Schythe". Anales Instituto Patagonia (Chile), Vol. 8: 391-392.

Zamora, E. 1978. Resumen Meteorológico año 1978. Estación Jorge C. Schythe. Anales Instituto Patagonia, (Chile) 9:231-236.

Zamora, E. \& A. Santana 1979. Oscilaciones y tendencias térmicas en Punta Arenas entre 1888-1979. Anales Instituto Patagonia (Chile), Vol. 10: 145-154.

Zamora, E \& A. Santana 1979. Resumen Meteorológico año 1979. Estación Jorge C. Schythe. Anales Instituto Patagonia, (Chile) 10:245-250.

Zamora, E \& A. Santana 1980. Resumen Meteorológico año 1980. Estación Jorge C. Schythe. Anales Instituto Patagonia, (Chile) 11:337-342.

Zamora, E \& A. Santana 1981. Resumen Meteorológico año 1981. Estación Jorge C. Schythe. Anales Instituto Patagonia, (Chile) 12:301-306. 